\title{
Statistical Approximation for Periodic Functions of Two Variables
}

\author{
Abdullah Alotaibi, ${ }^{1}$ M. Mursaleen, ${ }^{2}$ and S. A. Mohiuddine ${ }^{1}$ \\ ${ }^{1}$ Department of Mathematics, Faculty of Science, King Abdulaziz University, P.O. Box 80203, Jeddah 21589, Saudi Arabia \\ ${ }^{2}$ Department of Mathematics, Aligarh Muslim University, Aligarh 202002, India
}

Correspondence should be addressed to M. Mursaleen; mursaleenm@gmail.com

Received 4 October 2013; Accepted 17 November 2013

Academic Editor: Asadollah Aghajani

Copyright (C) 2013 Abdullah Alotaibi et al. This is an open access article distributed under the Creative Commons Attribution License, which permits unrestricted use, distribution, and reproduction in any medium, provided the original work is properly cited.

We prove a Korovkin type approximation theorem for a function of two variables by using the notion of statistical summability $(C, 1,1)$. We also study the rate of statistical summability $(C, 1,1)$ of positive linear operators. Finally we construct an example to show that our result is stronger than those previously proved for Pringsheim's convergence and statistical convergence.

\section{Introduction and Preliminaries}

In 1951, Fast [1] and Steinhaus [2] independently introduced an extension of the usual concept of sequential limit which is called statistical convergence.

The number sequence $x$ is said to be statistically convergent to the number $L$ provided that for each $\epsilon>0$,

$$
\lim _{n} \frac{1}{n}\left|\left\{k \leq n ;\left|x_{k}-\ell\right| \geq \epsilon\right\}\right|=0
$$

where $|\{k \leq n: k \in K\}|$ denotes the number of elements of $K$ not exceeding $n$. In this case we write $s t-\lim x_{k}=\ell$.

The notion of statistical convergence of double sequences $x=\left(x_{j k}\right)$ has been introduced and studied in $[3,4]$ independently in the same year, 2003.

Let $K \subseteq \mathbb{N} \times \mathbb{N}$ be a two-dimensional set of positive integers and let $K(n, m)$ be the numbers of $(i, j)$ in $K$ such that $i \leq n$ and $j \leq m$. Then the two-dimensional analogue of natural density can be defined as follows.

The lower asymptotic density of a set $K \subseteq \mathbb{N} \times \mathbb{N}$ is defined as

$$
\delta_{2}(K)=\lim _{n, m} \inf \frac{K(n, m)}{n m} .
$$

In this case the sequence $(K(n, m) / n m)$ has a limit in Pringsheim's sense then we say that $K$ has a double natural density and is defined as

$$
P-\lim \frac{K(n, m)}{n m}=\delta_{2}(K) .
$$

A real double sequence $x=\left(x_{j k}\right)$ is said to be statistically convergent to the number $l$ if for each $\epsilon>0$, the set

$$
\left\{(i, j), j \leq m, k \leq n:\left|x_{j k}-\ell\right| \geq \epsilon\right\}
$$

has double natural density zero. In this case we write $s t_{2}-\lim _{j, k} x_{j k}=\ell$.

If $x$ is statistically convergent, then $x$ need not be convergent. Also it is not necessarily bounded. For example, let $x=\left(x_{j k}\right)$ be defined as

$$
x_{j k}= \begin{cases}j k, & \text { if } j \text { and } k \text { are squares, } \\ 1, & \text { otherwise. }\end{cases}
$$

It is easy to see that $s t_{2}-\lim x_{j k}=1$, since the cardinality of the set $\left\{(j, k):\left|x_{j k}-1\right| \geq \epsilon\right\} \leq \sqrt{j} \sqrt{k}$ for every $\epsilon>0$. But $x$ is neither convergent nor bounded.

Móricz [5] introduced the notion of statistical summability $(C, 1,1)$. A double sequence $x=\left(x_{j k}\right)$ is said to be statistically summable $(C, 1,1)$ to the number $\ell$ if for every $\epsilon>$ 0 ,

$$
\delta_{2}\left\{(m, n) \in \mathbb{N} \times \mathbb{N}:\left|\sigma_{m n}-\ell\right| \geq \epsilon\right\}=0,
$$


where

$$
\sigma_{m n}=\frac{1}{(m+1)(n+1)} \sum_{j=0}^{m} \sum_{k=0}^{n} x_{j k}
$$

is the $(C, 1,1)$ mean of $x=\left(x_{j k}\right)$. Thus, the double sequence $x$ is statistically summable $(C, 1,1)$ to $l$ if and only if the sequence $\sigma=\left(\sigma_{m n}\right)$ is statistically convergent to $\ell$. In this case we write $s t_{2}(C, 1,1)-\lim _{j, k} x_{j k}=\ell$. Note that if a double sequence is bounded then $s t_{2}-\lim _{j, k} x_{j k}=\ell$ implies $s t_{2}-\lim _{m, n} \sigma_{m n}=\ell$.

Korovkin type approximation theorems (cf. [6-10]) are useful tools to check whether a given sequence $\left(L_{n}\right)_{n \geq 1}$ of positive linear operators on $C[0,1]$ of all continuous functions on the real interval $[0,1]$ is an approximation process. That is, these theorems exhibit a variety of test functions which assure that the approximation property holds on the whole space if it holds for them. Such a property was discovered by Korovkin in 1953 for test functions $1, x$, and $x^{2}$ in the space $C[0,1]$ as well as for test functions $1, \cos$, and $\sin$ in the space of all continuous $2 \pi$-periodic functions on the real line.

We know that $C[0,1]$ is a Banach space with norm

$$
\|f\|_{\infty}:=\sup _{x \in[0,1]}|f(x)|, \quad f \in C[0,1] .
$$

We denote by $C_{2 \pi}([0,1])$ the space of all $2 \pi$-periodic functions $f \in C([0,1])$ which is a Banach space with

$$
\|f\|_{2 \pi}=\sup _{t \in[0,1]}|f(t)| .
$$

After the paper of Gadjiev and Orhan [11], many papers have appeared in the literature concerning the Korovkin type approximation theorems via different statistical summability methods and for different sets of test functions. At present we are concerned about applications of such summability methods for double sequences to prove two-dimensional version of Korovkin theorem. For example, in [12,13] the authors used the notion of statistical $A$-summability of double sequences; in [13-16], the authors have used, respectively, statistical convergence and $A$-statistical convergence of double sequences; and in $[17,18]$, the authors used almost summability. For some more related work, we refer to [19-22].

In this paper, we present the Korovkin type approximation theorem for periodic functions via statistical summability $(C, 1,1)$ and also study the rate of statistical summability $(C, 1,1)$ of a double sequence of positive linear operators defined from $C^{*}\left(\mathbb{R}^{2}\right)$ into $C^{*}\left(\mathbb{R}^{2}\right)$, where $C^{*}\left(\mathbb{R}^{2}\right)$ is the space of all $2 \pi$-periodic and real valued continuous functions on $\mathbb{R}^{2}$ equipped with the norm

$$
\|f\|_{C\left(\mathbb{R}^{2}\right)}:=\sup _{(x, y) \in \mathbb{R}^{2}}|f(x, y)|, \quad\left(f \in C\left(\mathbb{R}^{2}\right)\right) .
$$

\section{Main Result}

First, we state the result due to [15] for $A$-statistical convergence of double sequences.
Theorem 1. Let $\left(L_{m n}\right)$ be a double sequence of positive linear operators acting from $C^{*}\left(\mathbb{R}^{2}\right)$ into $C^{*}\left(\mathbb{R}^{2}\right)$. Then, for all $f \in$ $C^{*}\left(\mathbb{R}^{2}\right)$

$$
s t_{2}^{A}-\lim _{m, n \rightarrow \infty}\left\|L_{m n}(f)-f\right\|_{C^{*}\left(\mathbb{R}^{2}\right)}=0
$$

if and only if

$$
s t_{2}^{A}-\lim _{m, n \rightarrow \infty}\left\|L_{m n}\left(f_{i}\right)-f_{i}\right\|_{C^{*}\left(\mathbb{R}^{2}\right)}=0, \quad i=0,1,2,3,4,
$$

where $f_{0}(x, y)=1, f_{1}(x, y)=\sin x, f_{2}(x, y)=\sin y, f_{3}(x$, $y)=\cos x$, and $f_{4}(x, y)=\cos y$.

If we replace the matrix $A$ by the identity four-dimensional matrix in the above theorem, then we immediately get the following result in Pringsheim's sense.

Corollary 2. Let $\left(L_{m n}\right)$ be a double sequence of positive linear operators acting from $C^{*}\left(\mathbb{R}^{2}\right)$ into $C^{*}\left(\mathbb{R}^{2}\right)$. Then, for all $f \in$ $C^{*}\left(\mathbb{R}^{2}\right)$

$$
P-\lim _{m, n \rightarrow \infty}\left\|L_{m n}(f)-f\right\|_{C^{*}\left(\mathbb{R}^{2}\right)}=0
$$

if and only if

$$
P-\lim _{m, n \rightarrow \infty}\left\|L_{m n}\left(f_{i}\right)-f_{i}\right\|_{C^{*}\left(\mathbb{R}^{2}\right)}=0, \quad i=0,1,2,3,4 .
$$

We prove the following result.

Theorem 3. Let $\left(T_{j k}\right)$ be a double sequence of positive linear operators acting from $C^{*}\left(\mathbb{R}^{2}\right)$ into $C^{*}\left(\mathbb{R}^{2}\right)$. Then, for all $f \in$ $C^{*}\left(\mathbb{R}^{2}\right)$

$$
s t_{2}(C, 1,1)-\lim _{j, k \rightarrow \infty}\left\|T_{j k}(f)-f\right\|_{C^{*}\left(\mathbb{R}^{2}\right)}=0
$$

if and only if

$$
\begin{array}{r}
s t_{2}(C, 1,1)-\lim _{j, k \rightarrow \infty}\left\|T_{j k}\left(f_{i}\right)-f_{i}\right\|_{C^{*}\left(\mathbb{R}^{2}\right)}=0 \\
(i=0,1,2,3,4) .
\end{array}
$$

Proof. Since each of the functions $f_{0}, f_{1}, f_{2}, f_{3}$, and $f_{4}$ belongs to $C^{*}\left(\mathbb{R}^{2}\right)$, necessity follows immediately from (15). Let condition (16) hold and $f \in C^{*}\left(\mathbb{R}^{2}\right)$. Let $I$ and $J$ be closed subintervals each of length $2 \pi$ of $\mathbb{R}$. $\operatorname{Fix}(x, y) \in I \times J$. By the continuity of $f$ at $(x, y)$, it follows that for given $\varepsilon>0$ there is a number $\delta>0$ such that for all $(u, v) \in \mathbb{R}^{2}$

$$
|f(u, v)-f(x, y)|<\varepsilon,
$$

whenever $|u-x|,|v-y|<\delta$. Since $f$ is bounded, it follows that

$$
|f(u, v)-f(x, y)| \leq M_{f}=\|f\|_{C^{*}\left(\mathbb{R}^{2}\right)},
$$

for all $(u, v) \in \mathbb{R}^{2}$.

For all $(u, v) \in(x-\delta, 2 \pi+x-\delta] \times(y-\delta, 2 \pi+y-\delta]$, it is well known that

$$
|f(u, v)-f(x, y)|<\varepsilon+\frac{2 M_{f}}{\sin ^{2}(\delta / 2)} \psi(u, v),
$$


where $\psi(u, v)=\sin ^{2}((u-x) / 2)+\sin ^{2}((v-y) / 2)$. Since the function $f \in C^{*}\left(\mathbb{R}^{2}\right)$ is $2 \pi$-periodic, the inequality (19) holds for $(u, v) \in \mathbb{R}^{2}$. Then, we obtain

$$
\begin{aligned}
\left|T_{j k}(f ; x, y)-f(x, y)\right| & \\
\leq & T_{j k}(|f(u, v)-f(x, y)| ; x, y) \\
+ & |f(x, y)|\left|T_{j k}\left(f_{0} ; x, y\right)-f_{0}(x, y)\right| \\
\leq & \left|T_{j k}\left(\varepsilon+\frac{2 M_{f}}{\sin ^{2}(\delta / 2)} \psi(u, v) ; x, y\right)\right| \\
+ & M_{f}\left|T_{j k}\left(f_{0} ; x, y\right)-f_{0}(x, y)\right| \\
\leq \varepsilon+ & \left(\left(\varepsilon+M_{f}\right)\left|T_{j k}\left(f_{0} ; x, y\right)-f_{0}(x, y)\right|\right) \\
+ & \frac{M_{f}}{\sin ^{2}(\delta / 2)} \\
\times & \left\{2\left|T_{j k}\left(f_{0} ; x, y\right)-f_{0}(x, y)\right|\right. \\
& +|\sin x|\left|T_{j k}\left(f_{1} ; x, y\right)-f_{1}(x, y)\right| \\
& +|\sin y|\left|T_{j k}\left(f_{2} ; x, y\right)-f_{3}(x, y)\right| \\
& +|\cos x|\left|T_{j k}\left(f_{3} ; x, y\right)-f_{3}(x, y)\right| \\
& \left.+|\cos y|\left|T_{j k}\left(f_{4} ; x, y\right)-f_{4}(x, y)\right|\right\} \\
<\varepsilon+ & K \sum_{i=0}^{4}\left|T_{j k}\left(f_{i} ; x, y\right)-f_{i}(x, y)\right|,
\end{aligned}
$$

where $K:=\varepsilon+M_{f}+\left(2 M_{f} / \sin ^{2}(\delta / 2)\right)$. Now, taking $\sup _{(x, y) \in I \times J}$, we get

$$
\left\|T_{j k}(f)-f\right\|_{C^{*}\left(\mathbb{R}^{2}\right)}<\varepsilon+K \sum_{i=0}^{4}\left\|T_{j k}\left(f_{i}\right)-f_{i}\right\|_{C^{*}\left(\mathbb{R}^{2}\right)} .
$$

Now for a given $r>0$ choose $\varepsilon^{\prime}>0$ such that $\varepsilon^{\prime}<r$. Define the following sets:

$$
\begin{aligned}
& D=\left\{(m, n):\left\|L_{m n}(f)-f\right\|_{C^{*}\left(\mathbb{R}^{2}\right)} \geq r\right\}, \\
& D_{i}=\left\{(m, n):\left\|L_{m n}\left(f_{i}\right)-f_{i}\right\|_{C^{*}\left(\mathbb{R}^{2}\right)} \geq \frac{r-\varepsilon^{\prime}}{5 K}\right\} \\
& (i=0,1,2,3,4),
\end{aligned}
$$

where $L_{m n}=(1 /(m+1)(n+1)) \sum_{j=0}^{m} \sum_{k=0}^{n} T_{j k}$. Then by $(21)$

$$
D \subseteq \bigcup_{i=0}^{4} D_{i}
$$

and so

$$
\delta_{A}^{(2)}(D) \leq \sum_{i=0}^{4} \delta_{A}^{(2)}\left(D_{i}\right)
$$

Now using (16), we get

$$
s t_{2}(C, 1,1)-\lim _{j, k \rightarrow \infty}\left\|T_{j k}(f)-f\right\|_{C^{*}\left(\mathbb{R}^{2}\right)}=0 .
$$

Example 4. Now we present an example of double sequences of positive linear operators, showing that Corollary 2 does not work but our approximation theorem works. We consider the double sequence of Fejer operators on $C^{*}\left(\mathbb{R}^{2}\right)$

$$
\begin{aligned}
\sigma_{m n}(f ; x, y)= & \frac{1}{(n \pi)} \cdot \frac{1}{(n \pi)} \\
& \times \int_{-\pi}^{\pi} \int_{-\pi}^{\pi} f(u, v) F_{m}(u) F_{n}(v) d u d v,
\end{aligned}
$$

where

$$
F_{m}(u)=\frac{\sin ^{2}(m(u-x) / 2)}{\sin ^{2}((u-x) / 2)}, \quad \frac{1}{\pi} \int_{-\pi}^{\pi} F_{m}(u) d u=1 .
$$

Observe that

$$
\begin{aligned}
& \sigma_{m n}\left(f_{0} ; x, y\right)=f_{0}(x, y), \\
& \sigma_{m n}\left(f_{1} ; x, y\right)=\frac{m-1}{m} f_{1}(x, y), \\
& \sigma_{m n}\left(f_{2} ; x, y\right)=\frac{n-1}{n} f_{2}(x, y), \\
& \sigma_{m n}\left(f_{3} ; x, y\right)=\frac{m-1}{m} f_{3}(x, y), \\
& \sigma_{m n}\left(f_{4} ; x, y\right)=\frac{n-1}{n} f_{4}(x, y) .
\end{aligned}
$$

Define a double sequence $\alpha=\left(\alpha_{m n}\right)$ by $\alpha_{m n}=(-1)^{m+n}$, $m, n \in \mathbb{N}$.

We observe that $\alpha=\left(\alpha_{m n}\right)$ is neither $P$-convergent nor statistically convergent but

$$
s t_{2}(C, 1,1)-\lim \alpha=0 .
$$

Let us define the operators $L_{m n}: C^{*}\left(\mathbb{R}^{2}\right) \rightarrow C^{*}\left(\mathbb{R}^{2}\right)$ by

$$
L_{m n}(f ; x, y)=\left(1+\alpha_{m n}\right) \sigma_{m n}(f ; x, y) .
$$

Then, observe that the double sequence of positive linear operators $\left(L_{m n}\right)$ defined by (30) satisfies all hypotheses of Theorem 3. Hence, by (28), we have, for all $f \in C^{*}\left(\mathbb{R}^{2}\right)$,

$$
s t_{2}(C, 1,1)-\lim _{m, n \rightarrow \infty}\left\|L_{m n}(f)-f\right\|_{C^{*}\left(\mathbb{R}^{2}\right)}=0 .
$$

Since $\left(\alpha_{m n}\right)$ is neither $P$-convergent nor statistically convergent, the sequence $\left(L_{m n}\right)$ given by (30) is also neither $P$ convergent nor statistically convergent to the function $f \in$ $C^{*}\left(\mathbb{R}^{2}\right)$. So, we conclude that Corollary 2 and Theorem 1 do not work for the operators $\left(L_{m n}\right)$ given by (30) while Theorem 3 still works. Hence, we conclude that $s t_{2}(C, 1,1)$ version is stronger than that of $P$-version as well as statistical version. 


\section{Rate of Statistical Summability $(C, 1,1)$}

Let $\left(\beta_{m n}\right)$ be a positive nonincreasing double sequence. We say that a double sequence $x=\left(x_{m n}\right)$ is statistically summable $(C, 1,1)$ to the number $L$ with the rate $o\left(\beta_{m n}\right)$ if for every $\varepsilon>0$,

$$
P-\lim _{m, n \rightarrow \infty} \frac{1}{\beta_{m n}}\left|\left\{j \leq m, k \leq n:\left|\sigma_{j k}-\ell\right| \geq \epsilon\right\}\right|=0
$$

In this case, we write $x_{m n}-L=s t_{2}(C, 1,1)-o\left(\beta_{m n}\right)$ as $m, n \rightarrow$ $\infty$.

Now, we recall the notion of modulus of continuity. The modulus of continuity of $f \in C^{*}\left(\mathbb{R}^{2}\right)$, denoted by $\omega(f, \delta)$ for $\delta>0$, is defined by

$$
\begin{gathered}
\omega(f, \delta)=\sup \left\{|f(u, v)-f(x, y)|:(u, v),(x, y) \in \mathbb{R}^{2},\right. \\
\left.\sqrt{(u-x)^{2}+(v-y)^{2}} \leq \delta\right\} .
\end{gathered}
$$

It is well known that

$$
\begin{aligned}
& |f(u, v)-f(x, y)| \\
& \quad \leq \omega\left(f, \sqrt{(u-x)^{2}+(v-y)^{2}}\right) \\
& \quad \leq \omega(f, \delta)\left(\frac{\sqrt{(u-x)^{2}+(v-y)^{2}}}{\delta}+1\right) .
\end{aligned}
$$

Then we have the following result.

Theorem 5. Let $\left(T_{j k}\right)$ be a double sequence of positive linear operators acting from $C^{*}\left(\mathbb{R}^{2}\right)$ into $C^{*}\left(\mathbb{R}^{2}\right)$. Let $\left(\alpha_{j k}\right)$ and $\left(\beta_{j k}\right)$ be two positive non-increasing sequences. Suppose that

(i) $\left\|T_{j k}\left(f_{0}\right)-f_{0}\right\|_{C^{*}\left(\mathbb{R}^{2}\right)}=s t_{2}(C, 1,1)-o\left(\alpha_{m n}\right)$,

(ii) $\omega\left(f, \lambda_{j k}\right)=s t_{2}(C, 1,1)-o\left(\beta_{j k}\right)$, where $\lambda_{m j k}=$ $\sqrt{\left\|T_{j k}(\varphi)\right\|_{C^{*}\left(\mathbb{R}^{2}\right)}}$ and

$$
\begin{gathered}
\varphi(u, v)=\sin ^{2}\left(\frac{u-x}{2}\right)+\sin ^{2}\left(\frac{v-y}{2}\right) \\
\text { for each }(u, v),(x, y) \in \mathbb{R}^{2} .
\end{gathered}
$$

Then, for all $f \in C^{*}\left(\mathbb{R}^{2}\right)$,

$$
\left\|T_{j k}(f)-f\right\|_{C^{*}\left(\mathbb{R}^{2}\right)}=s t_{2}(C, 1,1)-o\left(\gamma_{j k}\right),
$$

where $\gamma_{j k}=\max \left\{\alpha_{j k}, \beta_{j k}\right\}$.
Proof. Let $f \in C^{*}\left(\mathbb{R}^{2}\right)$ and $(x, y) \in-[\pi, \pi] \times[-\pi, \pi]$. Let $\delta>$ 0 ; we have the following cases.

Case I. If $\delta<|u-x| \leq \pi, \delta<|v-y| \leq \pi$, then $|u-x| \leq$ $\pi|\sin ((u-x) / 2)|$ and $|v-y| \leq \pi|\sin ((v-y) / 2)|$. Therefore by (34), we have

$$
\begin{aligned}
& |f(u, v)-f(x, y)| \\
& \quad \leq \omega(f, \delta)\left(\pi^{2} \frac{\sin ^{2}((u-x) / 2)+\sin ^{2}((v-y) / 2)}{\delta^{2}}+1\right) .
\end{aligned}
$$

Case II. If $|u-x|>\pi,|v-y| \leq \pi$. Let $k$ be an integer such that $|u+2 k \pi-x| \leq \pi$; then

$$
\begin{aligned}
& |f(u, v)-f(x, y)| \\
& =|f(u+2 k \pi, v)-f(x, y)| \\
& \leq \omega(f, \delta) \\
& \quad \times\left(\pi^{2} \frac{\sin ^{2}((u+2 k \pi-x) / 2)+\sin ^{2}((v-y) / 2)}{\delta^{2}}+1\right) \\
& =\omega(f, \delta)\left(\pi^{2} \frac{\sin ^{2}((u-x) / 2)+\sin ^{2}((v-y) / 2)}{\delta^{2}}+1\right) .
\end{aligned}
$$

Similarly, in the other two cases when $|u-x| \leq \pi,|v-y|>\pi$ and $|u-x|>\pi,|v-y|>\pi$, we obtain (37).

Now, using the definition of modulus of continuity and the linearity and the positivity of the operators $T_{j k}$, we get

$$
\begin{aligned}
& \left|T_{j k}(f ; x, y)-f(x, y)\right| \\
& \leq T_{j k}(|f(u, v)-f(x, y)| ; x, y) \\
& \quad+|f(x, y)|\left|T_{j k}\left(f_{0} ; x, y\right)-f_{0}(x, y)\right| \\
& \leq \omega(f, \delta) T_{j k}\left(f_{0} ; x, y\right)+\pi^{2} \frac{\omega(f, \delta)}{\delta^{2}} T_{j k}(\varphi ; x, y) \\
& \quad+|f(x, y)|\left|T_{j k}\left(f_{0} ; x, y\right)-f_{0}(x, y)\right| .
\end{aligned}
$$

Taking supremum over $(x, y)$ on both sides of the above inequality and let

$$
\delta:=\delta_{j k}=\sqrt{\left\|T_{j k}(\varphi)\right\|_{C^{*}\left(\mathbb{R}^{2}\right)}}
$$

We obtain

$$
\begin{aligned}
& \left\|T_{j k}(f)-f\right\|_{C^{*}\left(\mathbb{R}^{2}\right)} \\
& \leq \omega\left(f, \delta_{j k}\right)\left\|T_{j k}\left(f_{0}\right)-f_{0}\right\|_{C^{*}\left(\mathbb{R}^{2}\right)}+\left(1+\pi^{2}\right) \\
& \quad \times \omega\left(f, \delta_{j k}\right)+M\left\|T_{j k}\left(f_{0}\right)-f_{0}\right\|_{C^{*}\left(\mathbb{R}^{2}\right)},
\end{aligned}
$$


where $M:=\|f\|_{C^{*}\left(\mathbb{R}^{2}\right)}$. Let $L_{m n}=(1 /(m+1)(n+$ 1)) $\sum_{j=0}^{m} \sum_{k=0}^{n} T_{j k}$. Now for a given $\varepsilon>0$ define the following sets:

$$
\begin{aligned}
& D=\left\{(m, n):\left\|L_{m n}(f)-f\right\|_{C^{*}\left(\mathbb{R}^{2}\right)} \geq \varepsilon\right\}, \\
& D_{1}=\left\{(m, n):\left\|L_{m n}\left(f_{0}\right)-f_{0}\right\|_{C^{*}\left(\mathbb{R}^{2}\right)} \geq \frac{\varepsilon}{3}\right\}, \\
& D_{2}=\left\{(m, n): \omega\left(f, \delta_{m n}\right) \geq \frac{\varepsilon}{3\left(1+\pi^{2}\right)}\right\}, \\
& D_{3}=\left\{(m, n):\left\|L_{m n}(f)-f\right\|_{C^{*}\left(\mathbb{R}^{2}\right)} \geq \frac{\varepsilon}{3 M}\right\} .
\end{aligned}
$$

Then $D \subset D_{1} \cup D_{2} \cup D_{3}$. Further define

$$
\begin{aligned}
& D_{4}=\left\{(m, n): \omega\left(f, \delta_{m n}\right) \geq \sqrt{\frac{\bar{\varepsilon}}{3}}\right\}, \\
& D_{5}=\left\{(m, n):\left\|L_{m n}(f)-f\right\|_{C^{*}\left(\mathbb{R}^{2}\right)} \geq \sqrt{\frac{\bar{\varepsilon}}{3}}\right\} .
\end{aligned}
$$

We see that $D_{1} \subset D_{4} \cup D_{5}$. Therefore $D \subset \bigcup_{i=2}^{5} D_{i}$. Therefore, since $\gamma_{m n}=\max \left\{\alpha_{m n}, \beta_{m n}\right\}$, we conclude that for every $(j, k) \in \mathbb{N} \times \mathbb{N}$

$$
\delta_{2}(D) \leq \sum_{i=2}^{5} \delta_{2}\left(D_{i}\right)
$$

Using conditions (i) and (ii), we get $\left\|L_{m n}(f)-f\right\|_{C^{*}\left(\mathbb{R}^{2}\right)}=$ $s t_{2}(C, 1,1)-o\left(\gamma_{m n}\right)$.

\section{Acknowledgment}

The authors gratefully acknowledge the financial support from King Abdulaziz University, Jeddah, Saudi Arabia.

\section{References}

[1] H. Fast, "Sur la convergence statistique," Colloquium Mathematicum, vol. 2, pp. 241-244, 1951.

[2] H. Steinhaus, "Sur la convergence ordinaire et la convergence asymptotique," Colloquium Mathematicum, vol. 2, pp. 34-73, 1951.

[3] F. Móricz, "Statistical convergence of multiple sequences," Archiv der Mathematik, vol. 81, no. 1, pp. 82-89, 2003.

[4] M. Mursaleen and O. H. H. Edely, "Statistical convergence of double sequences," Journal of Mathematical Analysis and Applications, vol. 288, no. 1, pp. 223-231, 2003.

[5] F. Móricz, "Tauberian theorems for double sequences that are statistically summable $(C, 1,1)$," Journal of Mathematical Analysis and Applications, vol. 286, no. 1, pp. 340-350, 2003.

[6] F. Altomare, "Korovkin-type theorems and approximation by positive linear operators," Surveys in Approximation Theory, vol. 5, pp. 92-164, 2010.

[7] P. P. Korovkin, Linear Operators and Approximation Theory, Hindustan, New Delhi, India, 1960.

[8] O. H. H. Edely, S. A. Mohiuddine, and A. K. Noman, "Korovkin type approximation theorems obtained through generalized statistical convergence," Applied Mathematics Letters, vol. 23, no. 11, pp. 1382-1387, 2010.
[9] R. F. Patterson and E. Savaş, "Korovkin and Weierstrass approximation via lacunary statistical sequences," Journal of Mathematics and Statistics, vol. 1, no. 2, pp. 165-167, 2005.

[10] M. Mursaleen and A. Alotaibi, "Statistical summability and approximation by de la Vallée-Poussin mean," Applied Mathematics Letters, vol. 24, no. 3, pp. 320-324, 2011, Erratum: Applied Mathematics Letters, vol. 25, p. 665, 2012.

[11] A. D. Gadjiev and C. Orhan, "Some approximation theorems via statistical convergence," The Rocky Mountain Journal of Mathematics, vol. 32, no. 1, pp. 129-138, 2002.

[12] C. Belen, M. Mursaleen, and M. Yildirim, "Statistical A-summability of double sequences and a Korovkin type approximation theorem," Bulletin of the Korean Mathematical Society, vol. 49, no. 4, pp. 851-861, 2012.

[13] K. Demirci and S. Karakuş, "Korovkin-type approximation theorem for double sequences of positive linear operators via statistical $A$-summability," Results in Mathematics, vol. 63, no. 1-2, pp. 1-13, 2013.

[14] F. Dirik and K. Demirci, "Korovkin type approximation theorem for functions of two variables in statistical sense," Turkish Journal of Mathematics, vol. 34, no. 1, pp. 73-83, 2010.

[15] K. Demirci and F. Dirik, "Four-dimensional matrix transformation and rate of $A$-statistical convergence of periodic functions," Mathematical and Computer Modelling, vol. 52, no. 9-10, pp. 1858-1866, 2010.

[16] S. A. Mohiuddine and A. Alotaibi, "Statistical convergence and approximation theorems for functions of two variables," Journal of Computational Analysis and Applications, vol. 15, no. 2, pp. 218-223, 2013.

[17] G. A. Anastassiou, M. Mursaleen, and S. A. Mohiuddine, "Some approximation theorems for functions of two variables through almost convergence of double sequences," Journal of Computational Analysis and Applications, vol. 13, no. 1, pp. 37-46, 2011.

[18] S. A. Mohiuddine, "An application of almost convergence in approximation theorems," Applied Mathematics Letters, vol. 24, no. 11, pp. 1856-1860, 2011.

[19] S. A. Mohiuddine, A. Alotaibi, and M. Mursaleen, "Statistical summability $(C, 1)$ and a Korovkin type approximation theorem," Journal of Inequalities and Applications, vol. 2012, article 172, 2012.

[20] M. Mursaleen and A. Alotaibi, "Korovkin type approximation theorem for functions of two variables through statistical $A$ summability," Advances in Difference Equations, vol. 2012, article 65, 2012.

[21] M. Mursaleen and A. Alotaibi, "Korovkin type approximation theorem for statistical A-summability of double sequences," Journal of Computational Analysis and Applications, vol. 15, no. 6, pp. 1036-1045, 2013.

[22] C. Belen and S. A. Mohiuddine, "Generalized weighted statistical convergence and application," Applied Mathematics and Computation, vol. 219, no. 18, pp. 9821-9826, 2013. 


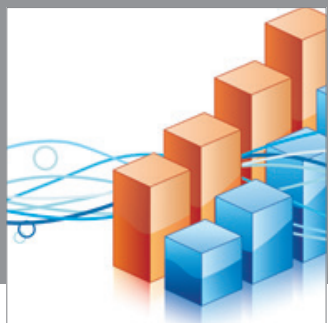

Advances in

Operations Research

mansans

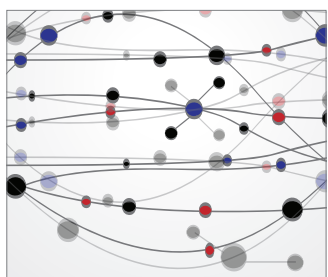

The Scientific World Journal
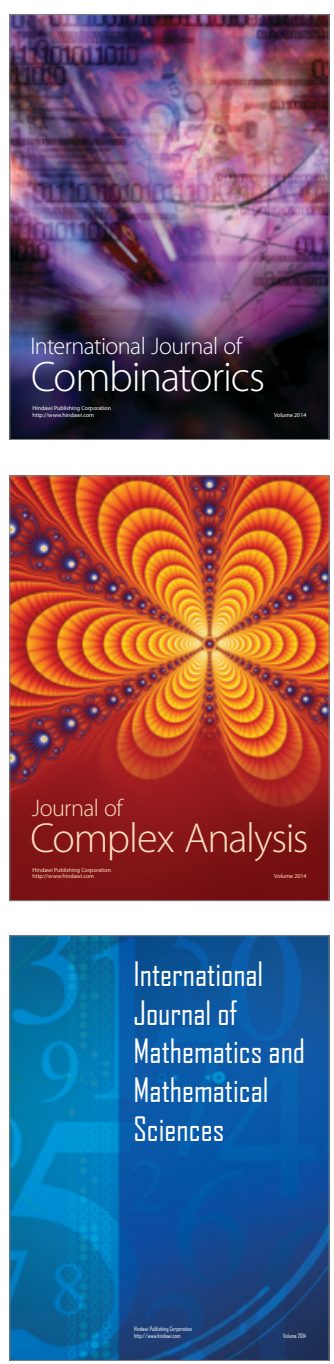
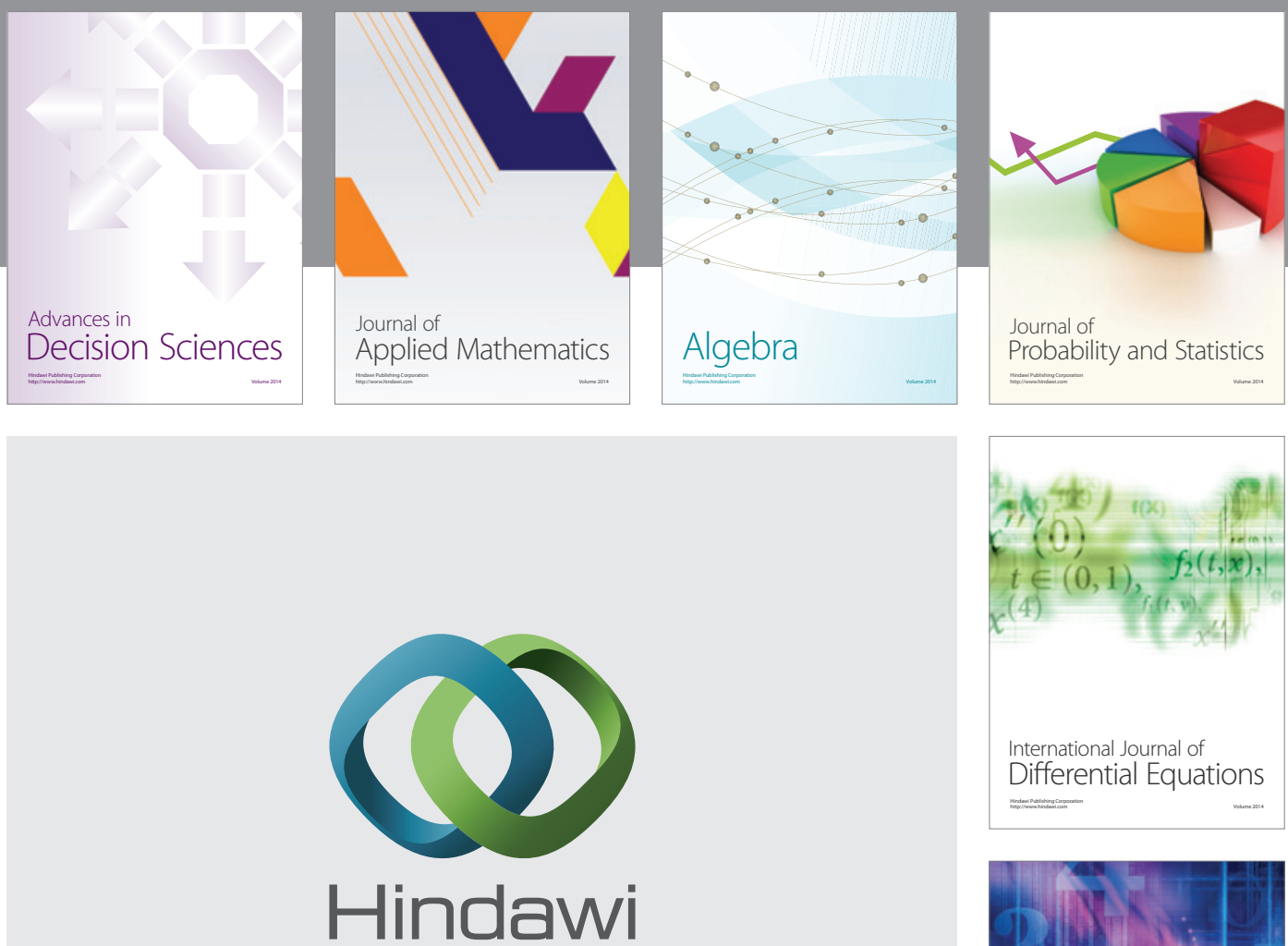

Submit your manuscripts at http://www.hindawi.com
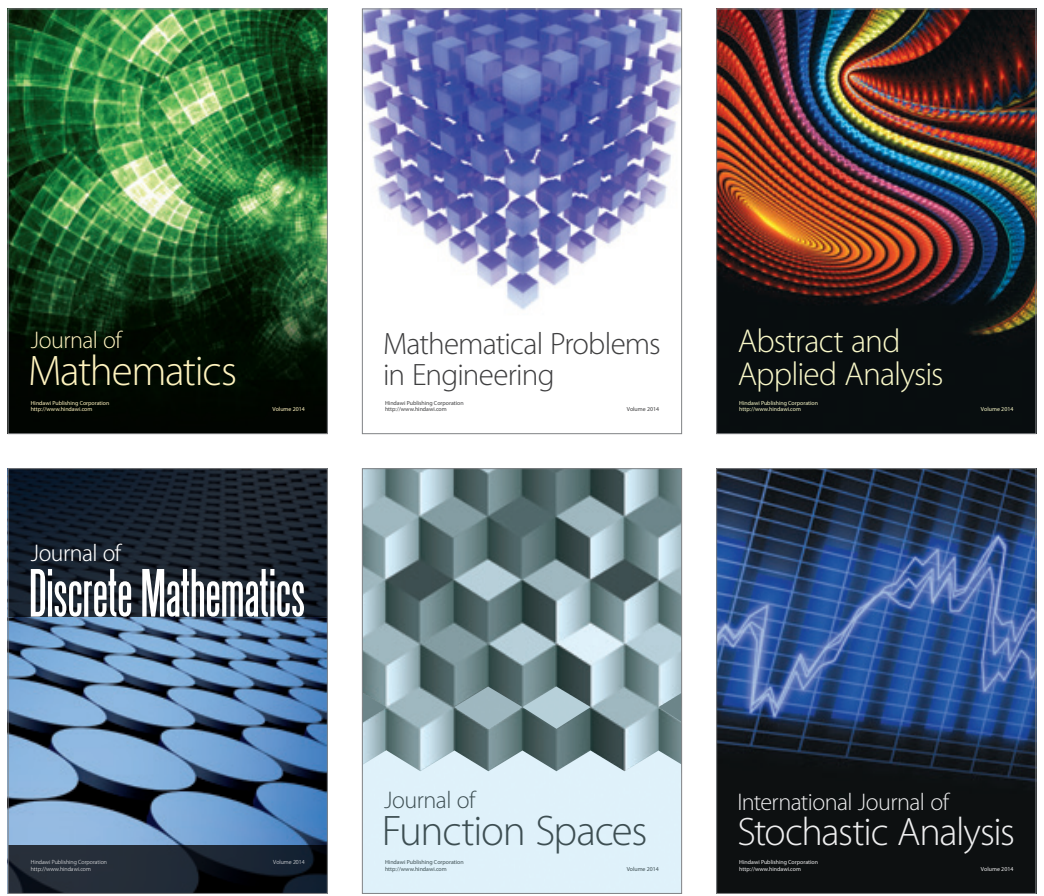

Journal of

Function Spaces

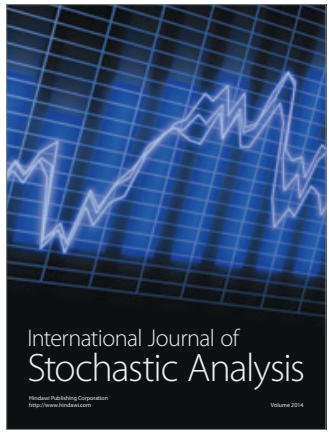

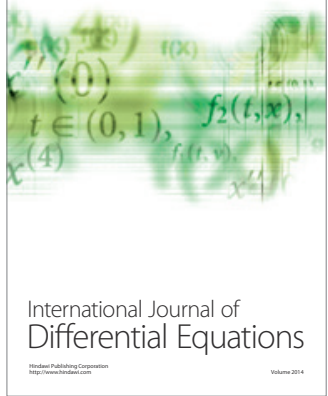
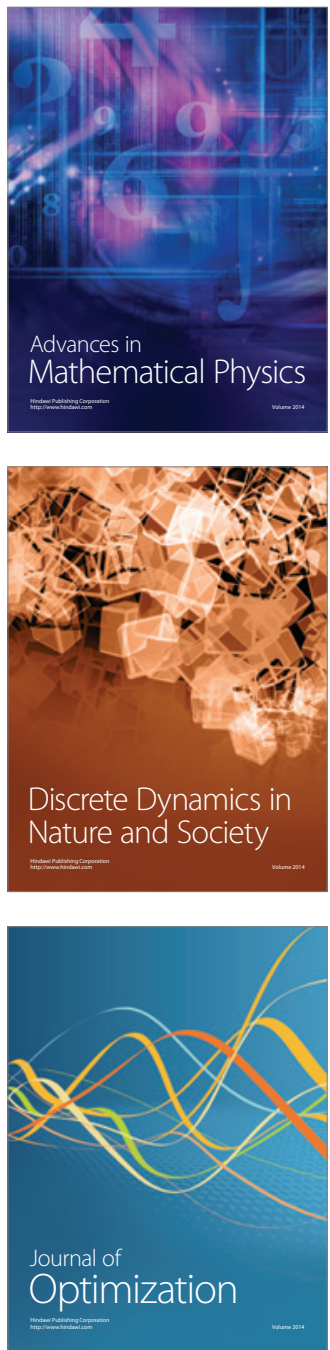